\title{
Development of a Quantum Dot-based Assay System for Detection of Specific HIV-I LTR Sequence Variants Asiedua Asante ${ }^{* \ddagger 1}$, Michael Nonnemacher ${ }^{1}$, Aikaterini Alexaki ${ }^{1}$, Elisabeth Papazoglou ${ }^{2}$, Peter Lelkes ${ }^{2}$ and Brian Wigdahl ${ }^{1}$
}

\author{
Address: ${ }^{1}$ Department of Microbiology and Immunology and Institute for Molecular Medicine and Infectious Disease, Philadelphia, PA, USA and \\ ${ }^{2}$ School of Biomedical Engineering, Science, and Health Systems, Drexel University College of Medicine, Philadelphia, PA, USA \\ * Corresponding author $¥$ Presenting author
}

from 2005 International Meeting of The Institute of Human Virology

Baltimore, USA, 29 August - 2 September 2005

Published: 8 December 2005

Retrovirology 2005, 2(SuppI I):P9 doi:I0.II86/I742-4690-2-SI-P9

Analysis of human immunodeficiency virus type 1 (HIV1) long terminal repeat (LTR) sequence variation within the CCAAT/enhancer binding protein (C/EBP) and stimulating protein (Sp) transcription factor binding sites has identified variants that correlate with HIV-associated dementia (HIVD). CdSe/ZnS nanocrytsals have facilitated the investigation of nano- and peco-scale biological components. Quantum dot-conjugated oligonucleotides homologous to specific variants of Sp site III and C/EBP site I, were utilized to quantitate the relative abundance of specific LTR variants. Quantum dot-conjugated oligonucleotides containing the Sp site III 5T binding site variant (C-to-T change at position 5) or the C/EBP site I 3T site variant, were reacted with plasmid DNA containing increasing concentrations of plasmid with the homologous LTR sequence variant. The results suggest that quantum dot-conjugated oligonucleotides specific for sequence variants within the LTR can be used as reporter molecules for identification and quantitation of HIV-1 genetic variation. 